\title{
Extracorporeal membrane oxygenator support in infants with systemic-pulmonary shunts
}

\author{
Phil Botha, PhD, FRCS, Shriprasad R. Deshpande, MBBS, MS, Michael Wolf, MD, Micheal Heard, RRT, \\ Bahaaldin Alsoufi, MD, Brian Kogon, MD, and Kirk Kanter, MD
}

\begin{abstract}
Background: Management of a patent systemic-pulmonary (SP) shunt and the resulting runoff during extracorporeal membrane oxygenation (ECMO) varies among institutions. We have used a strategy of increased flow without surgical reduction of the shunt diameter, and here report our results with this strategy.

Methods: In this database review of 169 successive veno-arterial ECMO runs performed between 2002 and 2013 in infants and neonates, ECMO flow, time to achieve lactate clearance, normal $\mathrm{pH}$, and negative fluid balance were compared in patients with shunts and those without shunts.
\end{abstract}

Results: Fifty-one of 169 infants $(30.2 \%)$ had a shunt in situ when ECMO was initiated. Significantly higher ECMO flows were maintained in the shunt group compared with the nonshunt group $(161 \pm 43 \mathrm{~mL} / \mathrm{kg} /$ minute vs $134 \pm 41 \mathrm{~mL} /$ $\mathrm{kg} /$ minute; $P<.001$ ). Infants with shunts had significantly higher pre-ECMO and peak lactate levels $(12.4 \pm 5.6 \mathrm{mmol} / \mathrm{L}$ vs $10.0 \pm 6.3 \mathrm{mmol} / \mathrm{L} ; P<.05$ and $13.7 \pm 4.9 \mathrm{mmol} / \mathrm{L}$ vs $11.6 \pm 5.5 \mathrm{mmol} / \mathrm{L} ; P<.02$, respectively) and required a longer period of support for clearance (median, 28.8 hours [16.1-63.3 hours] vs 17.5 hours [10.8-34.5 hours]; $P<.001)$. Although the absolute rate of lactate clearance was not significantly different between the 2 groups (median, $0.46 \mathrm{mmol} / \mathrm{L} /$ hour $[0.12-0.72 \mathrm{mmol} / \mathrm{L} /$ hour$]$ vs $0.48 \mathrm{mmol} / \mathrm{L} /$ hour [0.22-0.86 mmol/L/hour]; $P=.139$ ) the presence of a shunt, neonatal age, peak lactate, extracorporeal cardiopulmonary resuscitation, and the use of hemofiltration on ECMO significantly predicted the rate of clearance. Survival to hospital discharge was similar in the shunt and nonshunt groups $(49.0 \%$ vs $48.3 \% ; P=.932$ ).

Conclusions: A strategy of increased ECMO flow without surgically restricting shunt diameter appears to be successful in providing circulatory support in the majority of patients with an SP shunt. Equivalent survival suggests that routine surgical reduction of shunt diameter is not indicated. ( $\mathrm{J}$ Thorac Cardiovasc Surg 2016;152:912-8)

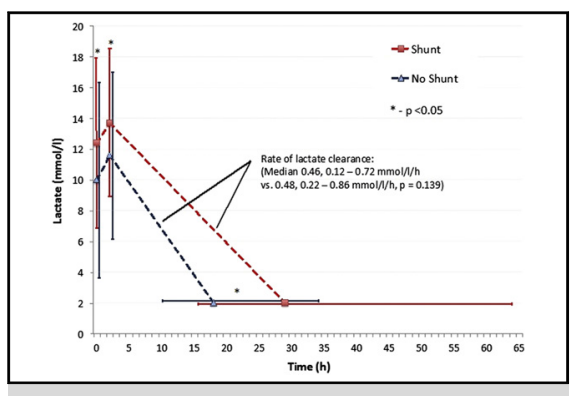

Variable clearance of lactic acid in shunt versus Sano patients on ECMO.

\section{Central Message}

A strategy of increased flow without restricting shunt flow may be adequate to achieve equivalent survival irrespective of the method of pulmonary blood flow (shunt vs Sano).

\section{Perspective}

Survival in single ventricle patients requiring ECMO support is poor. Strategies to maximize flow during ECMO successfully compensate without surgical restriction of shunt flow. There are subtle differences between the BlalockTaussig shunt and the Sano shunt; however, survival appears to be similar with the 2 types. Perioperative management and timing of escalation to ECMO warrant systematic investigation to improve overall outcomes.

See Editorial Commentary page 919.
Circulation in the presence of a surgical systemicpulmonary (SP) shunt is inherently unstable, particularly in the setting of single-ventricle physiology. Fluctuations in either systemic or pulmonary vascular tone can result in a profound and rapid alteration of systemic perfusion.

From Children's Healthcare of Atlanta, Emory University, Atlanta, Ga.

Received for publication July 15, 2015; revisions received March 26, 2016; accepted for publication March 30, 2016.

Address for reprints: Shriprasad R. Deshpande, MBBS, MS, Division of Pediatric Cardiology, Department of Pediatrics, Emory University School of Medicine,

Children's Healthcare of Atlanta, 1405 Clifton Rd, Atlanta, GA 30322-1101

(E-mail: deshpandes@kidsheart.com).

$0022-5223 / \$ 36.00$

Copyright (c) 2016 by The American Association for Thoracic Surgery

http://dx.doi.org/10.1016/j.jtcvs.2016.03.075
In the presence of systemic diastolic runoff into the low-resistance pulmonary bed, any reduction in the systemic perfusion pressure can lead to a rapid decompensation of myocardial perfusion and resulting impaired cardiac output. In addition to a tendency toward periods of pulmonary overcirculation, acute thrombosis of the shunt will similarly precipitate a profound collapse of the

Scanning this QR code will take you to the article title page. 


\section{Abbreviations and Acronyms \\ $\mathrm{BT}=$ Blalock-Taussig \\ $\mathrm{CVVH}=$ continuous veno-venous hemofiltration \\ $\mathrm{ECMO}=$ extracorporeal membrane oxygenation \\ $\mathrm{SP} \quad=$ systemic-pulmonary}

cardiovascular system. For these reasons, the clinician caring for patients with shunts is not infrequently faced with a need to support the collapsed circulation using extracorporeal membrane oxygenation (ECMO).

Initial reports of ECMO for postcardiotomy circulatory support considered patients with a systemic-pulmonary (SP) shunt unsuitable. The resultant runoff, increased pulmonary flow, and thus pulmonary venous return were thought to preclude successful support using this modality. ${ }^{1,2}$ Complete occlusion of the shunt by means of a surgical clip at the time of ECMO was shown to lead to unacceptable mortality rates, and thus many centers have adopted a strategy of increased flow to compensate for shunt runoff. ${ }^{3}$ It has been suggested that partial occlusion of the shunt during ECMO may reduce runoff into the pulmonary bed, resulting in improved perfusion pressure and hastening resolution of the malperfusion state. ${ }^{4}$ Our institution has pursued a strategy of liberal flow on ECMO without applying any limitation on shunt runoff. Here we review our results using this strategy in terms of resolution of the malperfusion state and other outcomes.

\section{METHODS}

We interrogated our prospectively collected ECMO database to identify all patients who underwent ECMO for cardiac indications in our institution between January 2002 and October 2013. These data were supplemented by chart review and examination of the electronic record of laboratory investigations. Neonates and infants who underwent veno-arterial ECMO with an SP shunt in situ (shunt group) were compared with infants and neonates who underwent veno-arterial ECMO without an SP shunt (nonshunt group) during the same period. Patients who underwent a first-stage Norwood procedure with a Sano right ventricle-pulmonary artery conduit were not included in the shunt group. In patients who underwent both precardiotomy and postcardiotomy ECMO, only the postoperative ECMO was analyzed.

The site of ECMO cannulation was dictated by the clinical situation. Cannulation was performed via central cannulation (reopening of the sternotomy incision) in the early postoperative period and via a neck incision (jugular and carotid vessels) in those requiring ECMO after 2 weeks postsurgery.

With cannulation via the right carotid artery, we aim to place the tip of the arterial cannula at the junction of the innominate artery and the aortic arch. This produces the possibility of occlusion of the systemic end of the shunt by the cannula, however. Nonetheless, we routinely continue ventilation in this setting, and a loss of end-tidal $\mathrm{CO}_{2}$ demonstrates shunt occlusion by the cannula. We have not observed this phenomenon, however. We further confirm the patency with routine echocardiography during ECMO, as well as during the weaning trials. Again, if there is any concern about cannula placement or shunt occlusion, the cannula is repositioned.

Similarly, for patients with an open chest poststernotomy, direct central cannulation was performed which involved cannulation of the right atrium (venous) and the aorta (arterial). ECMO was delivered using a standard circuit (S-97-E Tygon tubing; Medtronic, Minneapolis, Minn), oxygenator (pediatric Quadrox D; Maquet, Rastatt, Germany), and roller pump (Century, Mesa, Ariz) during this period. ECMO flows were increased to $\sim 100 \mathrm{~mL} / \mathrm{kg} /$ minute in the nonshunt group and up to $200 \mathrm{~mL} / \mathrm{kg} /$ minute in the shunt group. Flow rate was adjusted according to premembrane oxygen saturation and blood pressure.

Serum lactic acid level was measured using a regularly calibrated point-of-care analyzer (iSTAT, Abbott). The serum lactate level measured before the start of ECMO and the peak serum lactate measured during ECMO support were documented. Typically, serum lactate levels were measured initially approximately 2 hours in the early phase after institution of ECMO and according to clinical need thereafter. The time to lactate clearance was defined as the interval between the institution of ECMO and measurement of a serum lactate value $<2.0 \mathrm{mmol} / \mathrm{L}$ (the upper limit of normal in our laboratory). The rate of lactate clearance was calculated as peak lactate -2.0 , divided by the time elapsed between the start of ECMO and the first lactate measurement $<2.0 \mathrm{mmol} / \mathrm{L}$ recorded. For analysis of variables affecting the rate of lactate clearance, the range of clearance rates was divided into equal quartiles and entered into univariate and multivariate logistic regression models. For multivariate analysis, all variables with a $P$ value $<.10$ in univariate analysis were entered into the multinomial logistic regression model in a stepwise fashion. This analysis considered the highest quartile of lactate clearance as the reference category, and parameter estimates for the contrast with the lowest quartile are reported.

Arterial blood gas analysis was performed at hourly intervals initially after institution of ECMO using the same point-of-care analyzer as used for lactic acid measurements. The fluid balance was recorded in the electronic record throughout and analyzed in 12-hour intervals from 07:00 to 19:00 and from 19:00 to 07:00. The time at conclusion of the first 12-hour interval after the initiation of ECMO during which a negative fluid balance was achieved for that 12-hour period was taken as the time at which a negative fluid balance was attained for the analysis. Total fluid balance was calculated as total measured intake minus total measured fluid loss, not accounting for insensible losses.

Statistical analyses were performed using SPSS version 21 (IBM, Armonk, NY). Parametric continuous variables are presented as mean \pm standard deviation and analyzed using the Student $t$ test. Nonparametric variables are presented as median (interquartile range $[\mathrm{IQR}]$ ) and analyzed using the Wilcoxon rank-sum test or the Mann-Whitney $U$ test as indicated. In all analyses, a $P$ value $<.05$ was considered statistically significant. Our Institutional Review Board reviewed the study protocol and waived the requirement for individual informed consent. All coauthors have had full access to the data and have read and approved this final version of the manuscript.

\section{RESULTS}

During the period of January 2002 to October 2013, 218 patients underwent a total of 224 ECMO runs for cardiac indications. Of these, 169 patients were neonates or infants at the time of ECMO, including 51 with an SP shunt and 118 without a shunt. One patient with a diagnosis of pulmonary atresia/ventricular septal defect with a modified BlalockTaussig (BT) shunt placed during a previous admission underwent veno-venous ECMO for a suspected occluded shunt and thus was not included in the present analysis. Demographic data for the shunt and nonshunt groups are 
TABLE 1. Patient demographic data

\begin{tabular}{lccc}
\hline & $\begin{array}{c}\text { Nonshunt } \\
\text { (nunt group } \\
(\mathbf{n}=\mathbf{5 1})\end{array}$ & $\begin{array}{c}\text { group } \\
(\mathbf{n}=\mathbf{1 1 8})\end{array}$ & $\begin{array}{c}\boldsymbol{P} \\
\text { value }\end{array}$ \\
\hline Age, d, median (IQR) & $15(8-38)$ & $19(7-109)$ & NS \\
Weight, kg, median (IQR) & $3.3(2.7-3.8)$ & $3.6(2.9-5.4)$ & .010 \\
Male sex, n (\%) & $69(58.5)$ & $27(52.9)$ & NS \\
Diagnosis, n (\%) & & & \\
HLHS & $10(19.6)$ & $23(19.5)$ & \\
PA/IVS & $11(21.6)$ & $4(3.4)$ & \\
Other single ventricle & $22(43.1)$ & $11(9.3)$ & \\
TAPVR & 0 & $15(12.7)$ & \\
DCM/myocarditis & 0 & $11(9.3)$ & \\
TGA & $1(2.0)$ & $9(7.6)$ & \\
Truncus arteriosus & 0 & $8(6.8)$ & \\
Other & $7(13.7)$ & $37(31.4)$ & \\
Postoperative, n (\%) & $48(94.1)$ & $90(76.3)$ & .005 \\
Norwood stage 1, n (\%) & $16(31.4)$ & $28(23.7)$ & NS \\
ECPR, n (\%) & $37(72.5)$ & $56(47.5)$ & .003 \\
Shunt diameter, n (\%) & & & \\
3.0 mm & $8(15.7)$ & & \\
3.5 mm & $41(80.4)$ & & \\
4.0 mm & $2(3.9)$ & & \\
Indication for ECMO, n (\%) & & & \\
Inability to wean from CPB & $2(3.9)$ & $13(11.01)$ & \\
Cardiac arrest & $19(37.3)$ & $37(31.4)$ & \\
CV instability & $16(31.4)$ & $38+7(38.1)$ & \\
Hypoxia & $6(11.8)$ & $11(9.3)$ & \\
Respiratory arrest & $7(6.1)$ & $3(2.5)$ & \\
Concern for shunt thrombosis & $5(9.8)$ & 0 & \\
\hline
\end{tabular}

The shunt group comprises neonatal and infant patients requiring veno-arterial ECMO support during the study period. IQR, Interquartile range; $N S$, not significant; $H L H S$, hypoplastic left heart syndrome; PA/IVS, pulmonary atresia with intact ventricular septum; TAPVR, total anomalous pulmonary venous return; $D C M$, dilated cardiomyopathy; $T G A$, transposition of the great arteries; ECPR, extracorporeal cardiopulmonary resuscitation; ECMO, extracorporeal membrane oxygenation; $C P B$, cardiopulmonary bypass; $C V$, cardiovascular.

presented in Table 1. Patient age was not significantly different between the 2 groups (median, 15 days and 19 days, respectively; $P=.384$ ), but median infant weight was significantly lower in the shunt group (3.3 vs $3.6 \mathrm{~kg}$; $P<.010)$. A greater proportion of shunt patients underwent ECMO during the immediate postoperative admission $(94.1 \%$ vs $76.3 \% ; P<.005)$.

\section{ECMO Protocol}

ECMO was instituted in the operating room after failure to wean from cardiopulmonary bypass or for marginal hemodynamics after weaning in 20 patients in the nonshunt group $(16.9 \%)$ and 6 patients in the shunt group $(11.9 \%$; $P=.489)$. The remaining patients underwent cannulation in the cardiac intensive care unit. In the patients cannulated in the cardiac intensive care unit, the most common indication was cardiac arrest. Thus, these patients were resuscitated using the extracorporeal cardiopulmonary resuscitation (ECPR) pathway. A greater proportion of shunt patients compared with nonshunt patients received ECPR $(72.5 \%$ vs $47.5 \% ; P<.010)$. As shown in Table 1 , other indications included cardiovascular instability, hypoxia, respiratory arrest, and concern for shunt thrombosis. This distribution was comparable in the shunt and nonshunt groups. Decisions regarding cardiovascular instability were usually based on increasing needs for inotropic and vasopressor support, increased lactic acidosis, and other measures of low cardiac output, such as low near-infrared spectroscopy, poor perfusion, and narrow pulse pressure with tachycardia. This decision was made by the intensivist in conjunction with the primary surgeon.

The 5 patients who were cannulated owing to a concern for shunt thrombosis underwent diagnostic cardiac catheterization/angiography to evaluate shunt patency after stabilization. Shunt patency was established in each of these patients via angiography and or intervention as needed within 24 hours of initiation of ECMO. Thus, all 5 of these patients had a patent shunt through the remaining course of ECMO.

Cannulation was performed via the neck vessels in 10 patients in the shunt group $(19.6 \%)$ and in 33 patients in the nonshunt group $(28.0 \% ; P=.336)$, and via the open chest in the remaining patients. Significantly higher ECMO flows were maintained in the shunt group at 4 hours (mean, $161 \pm 43 \mathrm{~mL} / \mathrm{kg} /$ minute vs $134 \pm 41 \mathrm{~mL} / \mathrm{kg} /$ minute; $P<.001)$ and at 24 hours (mean, $148 \pm 43 \mathrm{~mL} / \mathrm{kg} /$ minute vs $124 \pm 38 \mathrm{~mL} / \mathrm{kg} /$ minute; $P<.01)$.

\section{Lactate Clearance}

Serum lactic acid levels immediately before ECMO were significantly higher in the shunt group compared with the nonshunt group (mean, $12.4 \pm 5.6 \mathrm{mmol} / \mathrm{L}$ vs $10.0 \pm 6.3 \mathrm{mmol} / \mathrm{L} ; P<.05)$. Similarly, peak lactate levels were significantly higher in the shunt group (mean, $13.7 \pm 4.9 \mathrm{mmol} / \mathrm{L}$ vs $11.6 \pm 5.5 \mathrm{mmol} / \mathrm{L} ; P<.02$ ).

Five patients ( 3 in the shunt group and 2 in the nonshunt group) died or were decannulated before lactate clearance could be achieved. A significantly greater proportion of patients in the shunt group required more than 48 hours to achieve lactate clearance $(31.4 \%$ vs $12.7 \% ; P=.004)$. The median time to achieve lactate clearance was significantly longer in the shunt group (28.8 hours [IQR, 16.1-63.3 hours] vs 17.5 hours [IQR, 10.8-34.5 hours]; $P<.001)$. Figure 1 shows the pre-ECMO and peak lactic acid levels, along with the median time to lactate clearance, in patients in the shunt and nonshunt groups requiring ECMO. Although the rate of lactate clearance was lower in the shunt group, the median difference did not reach statistical significance $(0.46 \mathrm{mmol} / \mathrm{L} / \mathrm{hour}$ [IQR, 0.12-0.72 $\mathrm{mmol} / \mathrm{L} /$ hour] vs $0.48 \mathrm{mmol} / \mathrm{L} / \mathrm{hour}$ [IQR, 0.22-0.86 mmol/L/hour]; $P=.139$ ). Results of univariate and multivariate analysis for factors affecting 


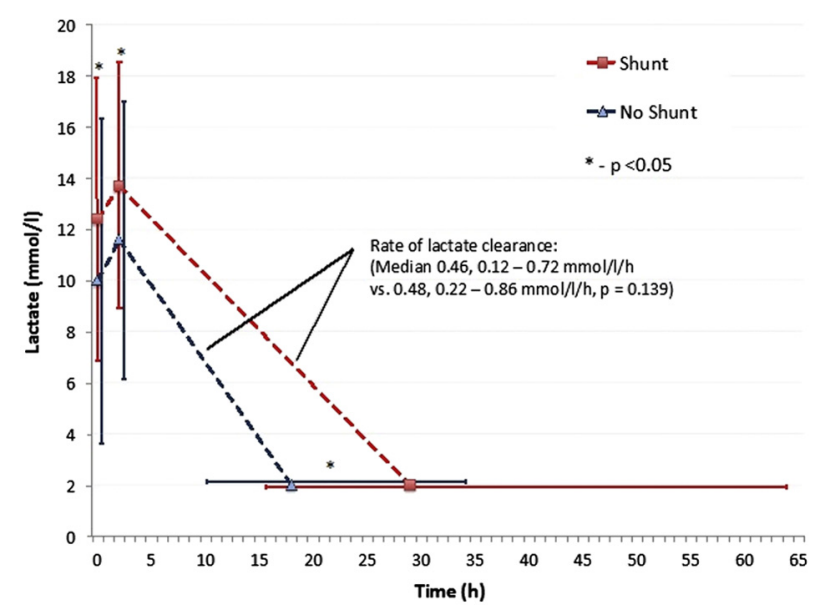

FIGURE 1. Line plot depicting mean serum lactic acid levels immediately before ECMO support and peak lactate level on ECMO, as well as the median time to achieve normal lactic acid levels in the shunt and nonshunt groups. Error bars depict SD for parametric variables and IQR for time to normalization of serum lactate level.

lactate clearance are listed in Table 2. In the multivariate model, peak serum lactic acid during ECMO, ECPR (as opposed to elective institution of ECMO), neonatal age, and the use of continuous veno-venous hemofiltration $(\mathrm{CVVH})$ were significant predictors of lactate clearance. The presence of an SP shunt was significantly predictive of being in the lowest quartile for rate of lactate clearance, but the interaction term size of shunt/body weight was not a statistically significant predictor. There was no relationship between the ECMO flow rate and clearance of lactate, independent of the presence or absence of an SP shunt.

\section{Acidemia/Fluid Balance}

The median lowest arterial blood $\mathrm{pH}$ recorded in the period immediately before ECMO cannulation or during ECMO was 7.120 (IQR, 7.022-7.225) in the shunt group and 7.183 (IQR, 7.054-7.244) in the nonshunt group, a non-statistically significant difference $(P=.074$, Mann-Whitney $U$ test). The time to achieve an arterial blood $\mathrm{pH}$ of $>7.350$ on ECMO also did not differ significantly between the shunt and nonshunt groups (median, 0.62 hours [IQR, 0.33-1.07 hours] vs 0.68 hours [IQR, 0.23-1.15 hours]; $P=.822$ ).

Data on fluid balance during ECMO were available for 24 patients in the shunt group and 70 patients in the nonshunt group. The median time to achieve a 12 -hour period of overall negative fluid balance was 47.0 hours (IQR, 34.6-59.2 hours) in the shunt group and 31.9 hours (IQR, 19.1-49.1 hours) in the nonshunt group $(P=.069)$. The overall fluid balance was not significantly different between the 2 groups, irrespective of normalization for the duration of ECMO (mean, $+24.6 \pm 657.3 \mathrm{~mL} /$ day vs $+48.9 \pm 888.4 \mathrm{~mL} /$ day; $P=.385)$.

\section{Norwood Stage 1 Procedure}

A prespecified subgroup analysis was performed with the cohort of patients who required ECMO support in the postoperative period after a stage 1 Norwood procedure. This cohort comprised 44 patients, $16(36 \%)$ who received a modified BT shunt and $28(64 \%)$ who received a Sano (right ventricle to pulmonary artery) shunt. The size of the BT shunt was $3 \mathrm{~mm}$ in 3 patients and $3.5 \mathrm{~mm}$ in 14 patients. Table 3 summarizes demographic and ECMO variables in these 2 subgroups. Patients in these 2 subgroups had similar mean weight, and a similar proportion underwent ECMO cannulation as part of an ECPR protocol. Pre-ECMO lactate and peak lactate values were not significantly different between the 2 subgroups. The median rate of lactate clearance during ECMO was lower than in the patients with a BT shunt compared with those with a Sano shunt $(0.29 \mathrm{mmol} / \mathrm{L} /$ hour [IQR, $0.08-0.56 \mathrm{mmol} / \mathrm{L} /$ hour] vs $0.47 \mathrm{mmol} / \mathrm{L} / \mathrm{hour} \quad[\mathrm{IQR}, \quad 0.28-0.82 \mathrm{mmol} / \mathrm{L} / \mathrm{hour}]$; $P=.052$, Mann-Whitney $U$ test).

\section{Outcomes}

The overall median duration of ECMO for the study subjects was 119.3 hours (IQR, 3.3-651.7 hours). The median duration of ECMO support was significantly longer in the nonshunt group compared with the shunt group (131.0 hours [IQR, 78.4-195 hours] vs 77.6 hours [IQR, 41.7-175 hours]; $P=.021$ ). Surrogate measures of low cardiac output or a malperfusion state, such as renal insufficiency, were also assessed as secondary endpoints. In addition to the fluid balance mentioned above (reflecting urine output), serum creatinine level and the need for CVVH post-ECMO were compared between the 2 groups. There was no difference in the serum creatinine values at decannulation or at the time of discharge. Only 2 patients, both in the shunt group, required CVVH post-ECMO, both of whom were on CVVH during the ECMO run. Owing to this very small number of patients, no further analysis related to persistent renal function post-ECMO was performed.

For the cohort of patients receiving ECMO support over this time period, survival to ECMO decannulation was $71.1 \%$, and survival to hospital discharge was $50.9 \%$. Survival for the entire study group was $48.5 \%$, and was not significantly different in the shunt and nonshunt groups $(49.0 \%$ vs $48.3 \% ; P=.932)$. Survival rates were nearly identical in the cohort of patients who underwent stage 1 Norwood procedure, $71.1 \%$ to ECMO decannulation and $46.7 \%$ to discharge.

\section{DISCUSSION}

Palliation of single-ventricle patients with a staged approach is now the standard accepted strategy. ${ }^{5}$ In conditions with inadequate pulmonary blood flow and those requiring single-ventricle palliation, for many patients the 
TABLE 2. Results of univariate and multivariate analyses for factors predictive of delayed lactate clearance

\begin{tabular}{|c|c|c|c|c|}
\hline & Univariate $P$ value & Multivariate $P$ value & Multivariate coefficient & $95 \% \mathrm{CI}$ \\
\hline Neonatal age & .013 & .029 & 4.291 & $1.160-15.866$ \\
\hline Weight (kg) & .111 & & & \\
\hline ECPR & $<.001$ & & & \\
\hline SP shunt & .174 & .019 & 4.926 & $1.303-18.681$ \\
\hline Shunt diameter/weight $(\mathrm{mm} / \mathrm{kg})$ & .409 & & & \\
\hline Pre-ECMO lactate & .012 & & & \\
\hline Peak lactate & $<.001$ & $<.001$ & 0.729 & $0.634-0.839$ \\
\hline CVVH & .006 & .050 & 0.280 & $0.079-0.999$ \\
\hline Stage 1 Norwood procedure & .344 & & & \\
\hline CPB time (min) & .148 & & & \\
\hline Crossclamp time (min) & .968 & & & \\
\hline Major bleeding & .158 & & & \\
\hline Neurologic event & .595 & & & \\
\hline
\end{tabular}

$\overline{C I}$, Confidence interval; $E C P R$, extracorporeal cardiopulmonary resuscitation; $S P$, systemic-pulmonary; $E C M O$, extracorporeal membrane oxygenation; $C V V H$, continuous veno-venous hemofiltration; $C P B$, cardiopulmonary bypass.

SP shunt still represents the first surgical step (or a part thereof). The period from the creation of the shunt until definitive repair or the substitution of the SP shunt with a cavopulmonary shunt remains the most critical phase in terms of risk for mortality and morbidity. ${ }^{6}$ The shunt-dependent circulation is characterized by lability, because the synthetic conduit used for the majority of such shunts does not allow for the usual degree of cardiovascular autoregulation. Creating a connection between the usually serial pulmonary and systemic circulations can lead to rapid and profound swings in perfusion in favor of either vascular bed.

Patient populations receiving SP shunts have changed considerably over the past 70 years, ${ }^{7,8}$ but the rate of in-hospital mortality has changed little, from $8.1 \%$ in the earliest reports ${ }^{9}$ to $4 \%$ to $10 \%$ in the higher-risk contemporary populations. ${ }^{8,10,11}$ Major morbidity also remains common with SP shunts, with reintervention required in $7.3 \%$ of recipients and $3 \%$ to $6 \%$ requiring ECMO support. ${ }^{11,12}$ We have analyzed a consecutive series of 169 infants and neonates requiring mechanical circulatory support in the form of veno-arterial ECMO, to ascertain the impact on outcomes of the presence of an SP shunt during ECMO support.

In early series of pediatric postcardiotomy ECMO, patients with shunts were considered unsuitable candidates because of the limitations imposed by shunt runoff on systemic perfusion. ${ }^{1,2}$ Jaggers and colleagues ${ }^{3}$ reported outcomes in 9 patients with shunts requiring ECMO. The authors occluded the shunt surgically at the time of ECMO in 4 patients, but had no survivors in this group. In contrast, in the 5 patients in whom the shunt was left

TABLE 3. Comparison of ECMO conduct and metabolic normalization in first-stage Norwood patients undergoing ECMO between the subset with systemic-pulmonary shunts and those with right ventricle-to-pulmonary artery conduits

\begin{tabular}{|c|c|c|c|}
\hline Variable & BT shunt group $(\mathrm{n}=16)$ & Sano group $(\mathbf{n}=28)$ & $P$ value \\
\hline Weight, $\mathrm{kg}$, mean $\pm \mathrm{SD}$ & $3.28 \pm 0.67$ & $3.11 \pm 0.67$ & .493 \\
\hline $\mathrm{ECPR}, \mathrm{n}(\%)$ & $9(56)$ & $17(61)$ & .954 \\
\hline \multicolumn{4}{|l|}{ Indication for ECMO, n (\%) } \\
\hline Inability to wean from CPB & $2(13)$ & $2(7)$ & \\
\hline Cardiac arrest & $2(13)$ & $12(43)$ & \\
\hline CV instability & $5(31)$ & $11(39)$ & \\
\hline Hypoxia & $1(6)$ & $2(7)$ & \\
\hline Respiratory arrest & 0 & $1(4)$ & \\
\hline Metabolic & $2(13)$ & 0 & \\
\hline Shunt thrombosis & $4(25)$ & 0 & \\
\hline Pre-ECMO lactate, mmol/L, mean $( \pm \mathrm{SD})$ & $13.0(5.2)$ & $11.4(5.1)$ & .362 \\
\hline Peak lactate, mmol/L, mean $( \pm \mathrm{SD})$ & $13.2(4.4)$ & $13.1(4.9)$ & .407 \\
\hline Lactate clearance rate, $\mathrm{mmol} / \mathrm{L} / \mathrm{h}$, mean $( \pm \mathrm{SD})$ & $0.29(0.32)$ & $0.47(0.41)$ & .052 \\
\hline Time to $\mathrm{pH}>7.35, \mathrm{~h}$, mean $( \pm \mathrm{SD})$ & $0.63(0.46)$ & $0.71(2.36)$ & .464 \\
\hline Time to negative fluid balance, $\mathrm{h}$, mean $( \pm \mathrm{SD})$ & $45.7(20.5)$ & $28.6(20.95)$ & .185 \\
\hline Total fluid balance, $\mathrm{mL} / \mathrm{d}$, mean $( \pm \mathrm{SD})$ & $-116.1(430.9)$ & $2.0(310.0)$ & .129 \\
\hline
\end{tabular}

Significant $P$ values are in bold type. $B T$, Blalock-Taussig; $S D$, standard deviation; $E C P R$, extracorporeal cardiopulmonary resuscitation; $E C M O$, extracorporeal membrane oxygenation; $C P B$, cardiopulmonary bypass; $C V$, cardiovascular. 
patent and the ECMO flow increased to compensate for shunt runoff, mortality was considerably lower, at $20 \%$. More recently, Allan and colleagues ${ }^{4}$ evaluated 44 patients who underwent ECMO following single-ventricle palliation with a SP shunt. ${ }^{4}$ The authors maintained shunt patency wherever possible, but in patients exhibiting decreased systemic perfusion with delayed clearance of lactate despite increasing ECMO flow, placed 1 or more surgical clips to restrict the shunt diameter and reduce runoff. This intervention was required in 17 of the 44 patients $(39 \%)$. Overall survival to hospital discharge was $48 \%$, with $20 \%$ of survivors requiring shunt clipping, as opposed to $61 \%$ of nonsurvivors. In the present series, our patients in the shunt group requiring ECMO had lower body weight and required ECMO predominantly in the postoperative period, most commonly as part of extracorporeal resuscitation for cardiovascular collapse. Significantly higher ECMO flows were maintained in the shunt group, and no surgical reduction of shunt diameter was undertaken. Survival to discharge in all infants and neonates requiring ECMO in our series was comparable to that reported in previous studies and was not affected by the presence of an SP shunt.

As a surrogate measure of tissue oxygen delivery and anaerobic metabolism, serum lactic acid level at various postoperative time points has been shown to be predictive of outcomes in pediatric cardiac surgery. ${ }^{13-15}$ Several factors determine the level of serum lactate in an infant requiring ECMO. The duration and severity of the low cardiac output/hypoxemic state prompting the need for ECMO are major determinants, but other factors, including duration of cardiopulmonary bypass, circulatory arrest, and/or regional perfusion, also have been shown to correlate with early postoperative serum lactate level. ${ }^{16,17}$ Because the patients in the shunt group in our series were usually in the perioperative period and more likely to have required ECMO after cardiac arrest and thus as part of an extracorporeal cardiopulmonary resuscitation protocol, we found, not surprisingly, significantly higher pre-ECMO serum lactic acid levels in this group. Multiple factors determine the rate of lactate clearance in patients during ECMO as well. Whether or not the precipitant of cardiovascular collapse has resolved will have a profound effect, as will the presence of residual uncorrected anatomic abnormalities. The adequacy of organ perfusion during ECMO will determine the level of ongoing lactate production and the rate at which the existing pool will be metabolized. Although the rate of lactate clearance in our shunt group patients appeared to be lower, the median difference in the rate of clearance on ECMO did not reach statistical significance. On multivariate analysis, peak serum lactic acid during ECMO, ECPR, neonatal age, and the use of CVVH were significant predictors of the rate of lactate clearance. The presence of an SP shunt was also a significant predictor of being in the lowest quartile of lactate clearance rate, but the relative size of the shunt did not appear to be significant.

It has been a concern that achieving and maintaining higher ECMO flows in patients with a patent SP shunt may come at the expense of a greater need for volume administration, and thus delay the resolution of fluid sequestration. The median time to achieving a 12-hour period of overall negative fluid balance was 47.0 hours (IQR, 34.6-59.2 hours) in the shunt group versus 31.9 hours (IQR, 19.1-49.1 hours) in the nonshunt group. Although numerous factors could have influenced this trend, including lower renal perfusion and a higher requirement for $\mathrm{CVVH}$ in the shunt group, the difference did not reach statistical significance $(P=.069)$. The lower number of patients for whom all of the required datapoints were available raises the possibility of a type 2 error in this analysis.

We also analyzed, as a more homogenous subgroup, all patients who required ECMO after a stage 1 Norwood procedure, comparing those with a BT shunt and those with a Sano shunt. Although pre-ECMO and peak serum lactate levels were not significantly different between these 2 groups, the patients with a BT shunt had a significantly lower rate of lactate clearance. Polimenakos and colleagues $^{18}$ reported on 20 functional single- ventricle patients supported with ECMO after cardiotomy. They left the shunt patent in all patients in the hope of minimizing pulmonary vascular endothelial injury and used higher pump flows ( $>150 \mathrm{~mL} / \mathrm{kg} / \mathrm{minute}$ ) to compensate for shunt runoff. Vasoactive-inotropic support was continued and 20-ppm inhaled nitric oxide was administered in all patients. Twelve of these patients had pulmonary blood flow established via a Sano shunt, which likely would have had significant less impact on flow requirement. Survival to discharge was $57 \%$. Ravishankar and colleagues ${ }^{19}$ reported a $39 \%$ rate of survival to discharge in 36 neonates rescued by ECMO following stage 1 palliation. They noted that the shunt was left patent (a modified BT shunt in the majority of cases), requiring a flow rate of 150 to $200 \mathrm{~mL} / \mathrm{kg} /$ minute to compensate for shunt runoff. Similarly, in an analysis from the ELSO registry, ${ }^{20}$ in patients with hypoplastic left heart syndrome who received stage 1 palliation requiring ECMO, 59\% survived the decannulation, and $31 \%$ survived to discharge. The risk factors associated with mortality were black race, lower weight, and longer duration of ventilator support before ECMO. However, the authors were unable to comment on the type of shunt (SP shunt vs Sano shunt), other surgical details (eg, clipped shunts), or patient acuity before ECMO. In our experience, despite a slower lactate clearance rate in patients with an SP shunt, survival to discharge was not affected irrespective of the shunt type, and was uniformly better ( $48 \%$ ) than that published in the ELSO registry report. 


\section{Limitations}

The study is inherently limited in generalizability by its retrospective nature and small sample size. Although a standardized protocol for the management of all patients on ECMO was followed, this underwent some evolution over the 11-year time course of the study.

\section{CONCLUSIONS}

Mortality remains common in infants requiring support with ECMO. Accepting the limitations of mortality as an endpoint, the presence of an SP shunt during ECMO support does not appear to have an adverse effect on outcome, if ECMO flow is appropriately increased to compensate for shunt runoff. Although patients with a patent SP shunt appeared to have delayed resolution of the malperfusion state on multivariate analysis, our data do not support routine surgical reduction of shunt diameter to reduce shunt runoff.

\section{Conflict of Interest Statement}

Authors have nothing to disclose with regard to commercial support.

\section{References}

1. Kanter KR, Pennington G, Weber TR, Zambie MA, Braun P, Martychenko V. Extracorporeal membrane oxygenation for postoperative cardiac support in children. J Thorac Cardiovasc Surg. 1987;93:27-35.

2. del Nido PJ, Dalton HJ, Thompson AE, Siewers RD. Extracorporeal membrane oxygenator rescue in children during cardiac arrest after cardiac surgery. Circulation. 1992;86(5 Suppl):II300-4.

3. Jaggers JJ, Forbess JM, Shah AS, Meliones JN, Kirshbom PM, Miller CE, et al Extracorporeal membrane oxygenation for infant postcardiotomy support: significance of shunt management. Ann Thorac Surg. 2000;69:1476-83.

4. Allan CK, Thiagarajan RR, del Nido PJ, Roth SJ, Almodovar MC, Laussen PC. Indication for initiation of mechanical circulatory support impacts survival of infants with shunted single-ventricle circulation supported with extracorporeal membrane oxygenation. J Thorac Cardiovasc Surg. 2007;133: 660-7.

5. Williams JA, Bansal AK, Kim BJ, Nwakanma LU, Patel ND, Seth AK, et al. Two thousand Blalock-Taussig shunts: a six-decade experience. Ann Thorac Surg. 2007;84:2070-5.

6. Fenton KN, Siewers RD, Rebovich B, Pigula FA. Interim mortality in infants with systemic-to-pulmonary artery shunts. Ann Thorac Surg. 2003;76:152-6.
7. Blalock A, Taussig HB. Landmark article, May 19, 1945. The surgical treatment of malformations of the heart in which there is pulmonary stenosis or pulmonary atresia. JAMA. 1984;251:2123-38.

8. Heidari-Bateni G, Norouzi S, Hall M, Brar A, Eghtesady P. Defining the best practice patterns for the neonatal systemic-to-pulmonary artery shunt procedure. J Thorac Cardiovasc Surg. 2014;147:869-73.e3.

9. de Leval MR, McKay R, Jones M, Stark J, Macartney FJ. Modified BlalockTaussig shunt: use of subclavian artery orifice as flow regulator in prosthetic systemic-pulmonary artery shunts. J Thorac Cardiovasc Surg. 1981;81:112-9.

10. Myers JW, Ghanayem NS, Cao Y, Simpson P, Trapp K, Mitchell ME, et al. Outcomes of systemic to pulmonary artery shunts in patients weighing less than $3 \mathrm{~kg}$ : analysis of shunt type, size, and surgical approach. J Thorac Cardiovasc Surg. 2014;147:672-7.

11. Petrucci O, O'Brien SM, Jacobs ML, Jacobs JP, Manning PB, Eghtesady P. Risk factors for mortality and morbidity after the neonatal Blalock-Taussig shunt procedure. Ann Thorac Surg. 2011;92:642-51.

12. Guzzetta NA, Foster GS, Mruthinti N, Kilgore PD, Miller BE, Kanter KR. In-hospital shunt occlusion in infants undergoing a modified Blalock-Taussig shunt. Ann Thorac Surg. 2013;96:176-82.

13. Siegel LB, Dalton HJ, Hertzog JH, Hopkins RA, Hannan RL, Hauser GJ. Initial postoperative serum lactate levels predict survival in children after open heart surgery. Intensive Care Med. 1996;22:1418-23.

14. Charpie JR, Dekeon MK, Goldberg CS, Mosca RS, Bove EL, Kulik TJ. Serial blood lactate measurements predict early outcome after neonatal repair or palliation for complex congenital heart disease. J Thorac Cardiovasc Surg. 2000; 120:73-80.

15. Schumacher KR, Reichel RA, Vlasic JR, Yu S, Donohue J, Gajarski RJ, et al. Rate of increase in serum lactate level risk-stratifies infants after surgery for congenital heart disease. J Thorac Cardiovasc Surg. 2014;148:589-95.

16. Munoz R, Laussen PC, Palacio G, Zienko L, Piercey G, Wessel DL. Changes in whole blood lactate levels during cardiopulmonary bypass for surgery for congenital cardiac disease: an early indicator of morbidity and mortality. J Thorac Cardiovasc Surg. 2000;119:155-62.

17. Algra SO, Schouten AN, van Oeveren W, van der Tweel I, Schoof PH, Jansen NJ, et al. Low-flow antegrade cerebral perfusion attenuates early renal and intestinal injury during neonatal aortic arch reconstruction. J Thorac Cardiovasc Surg. 2012;144:1323-8.

18. Polimenakos AC, Wojtyla P, Smith PJ, Rizzo V, Nater M, El Zein CF, et al. Post-cardiotomy extracorporeal cardiopulmonary resuscitation in neonates with complex single ventricle: analysis of outcomes. Eur J Cardiothorac Surg. 2011;40:1396-405.

19. Ravishankar C, Dominguez TE, Kreutzer J, Wernovsky G, Marino BS, Godinez R, et al. Extracorporeal membrane oxygenation after stage I reconstruction for hypoplastic left heart syndrome. Pediatr Crit Care Med. 2006;7:319-23.

20. Sherwin ED, Gauvreau K, Scheurer MA, Rycus PT, Salvin JW, Almodovar MC, et al. Extracorporeal membrane oxygenation after stage 1 palliation for hypoplastic left heart syndrome. J Thorac Cardiovasc Surg. 2012;144:1337-43.

Key Words: aorto-pulmonary shunt, congenital heart disease, extracorporeal membrane oxygenation, pediatric, univentricular heart 